\title{
La figura abyecta del mestizo en El primer nueva corónica y buen gobierno
}

\author{
OLIMPIA E. ROSENTHAL \\ Indiana University-Bloomington \\ orosenth@indiana.edu

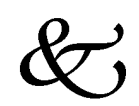

Resumen

En el presente ensayo analizo la caracterización del mestizo en El primer nueva corónica y buen gobierno de Felipe Guamán Poma de Ayala. Se argumenta que el autor intenta evadir toda representación sistemática sobre esta figura y se plantea que es posible detectar dos propuestas concretas alrededor de las cuales Guamán Poma organiza sus comentarios sobre mestizos. Por un lado, insiste en la necesidad de segregar a la comunidad amerindia en sus pueblos de indios para apartarla de todo contacto con otros grupos socio-raciales y así evitar la proliferación de mestizos. Por otro lado, Guamán Poma identifica la sexualidad femenina amerindia como un ámbito crucial de intervención para desalentar la reproducción de este grupo. Se propone, además, que el mestizo aparece en la obra de Guamán Poma no como un componente más de su meticulosa representación de la sociedad colonial, sino más bien como la figura de lo abyecto.

Palabras claves: Felipe Guamán Poma de Ayala, mestizos, abyecto, segregación, reproducción, sexualidad femenina amerindia.

\begin{abstract}
In this article I analyze the characterization of mestizos in Guamán Poma's magnum opus. I argue that the author evades any type of systematic representation of this group and, instead, organizes his comments about mestizos around two interrelated proposals. On the one hand, he insists on the need to segregate the indigenous from other groups in order to prevent the proliferation of mestizos. On the other hand, he identifies Amerindian female sexuality as a key site of intervention
\end{abstract}


OLIMPIA E. ROSENTHAL

for deterring the reproduction of this group. I suggest, moreover, that mestizos appear in this text as the figure of the abject.

Keywords: Felipe Guamán Poma de Ayala, mestizos, abject, segregation, reproduction, Amerindian female sexuality.

Recibido: 1/11/14 Aceptado: 18/11/14 Publicado on line: 10/1/15

Uno de los aspectos más sobresalientes de El primer nueva corónica y buen gobierno, de Felipe Guamán Poma de Ayala, es la caracterización abyecta de la figura del mestizo. Salvo en casos excepcionales como el de su medio hermano Martín de Ayala o el del minero Juan García de la Vega (a quienes presenta en términos positivos), Guamán Poma describe categóricamente a los mestizos como una "casta maldita," compuesta de borrachos, soberbios, malos cristianos y enemigos de los indígenas (pp. 18, 748, 448, 551). Asimismo, denuncia abiertamente las relaciones matrimoniales con mestizos -en ocasiones apelando para esto a nociones de pureza de sangre-, critica vehementemente la reproducción de este grupo, e insiste en la segregación total entre Amerindios y el resto de la población colonial (Guamán Poma de Ayala, 1615/1616, pp. 510, 215, 448). Como arguye Rolena Adorno (2000), la reprobación contundente del mestizo que encontramos en la carta-crónica de Guamán Poma constituye una de las premisas básicas en las que se funda la conceptualización de esta obra (p. xli). Lo sorprendente, por ende, es que este aspecto del texto haya recibido tan poca atención crítica. El objetivo central del presente ensayo es precisamente contribuir al desarrollo crítico-analítico de esta cuestión.

Comienzo analizando la manera en que Guamán Poma estructura sus comentarios sobre mestizos y sugiero que, tanto en la forma como en el contenido del texto, el autor busca evadir cualquier tipo de representación sistemática sobre el mestizaje en general y sobre la figura del mestizo en particular. En vez de llevar a cabo tal representación, argumento que Guamán Poma organiza las observaciones que hace sobre mestizos alrededor de dos propuestas concretas cuyo último fin es repudiar la continua reproducción de este grupo. Por un lado, insiste en la necesidad de segregar a la comunidad amerindia en sus pueblos de indios para apartarla de todo contacto con otros grupos socio-raciales y así evitar la creciente proliferación de mestizos. Por otro lado, Guamán Poma exhorta a las mujeres indígenas a preservar su castidad, recalca la importancia de la reproducción endogámica y propone la protección de la sexualidad femenina amerindia como parte de las reformas coloniales que plantea. Es decir, identifica la sexualidad femenina amerindia como un ámbito crucial de intervención para frenar la reproducción de mestizos. A lo largo del presente análisis, propongo que el mestizo aparece en la obra de Guamán Poma no como un componente más de su meticulosa representación 
de la sociedad colonial, sino más bien como la figura de lo abyecto; es decir, como aquello que cuestiona toda noción de fronteras estables y cuya exclusión es constitutiva de la identidad del sujeto indígena al que busca representar y proteger. ${ }^{1}$

La figura del mestizo -a pesar de ser evocada más de cincuenta veces por escrito y tres veces por medio de visuales- nunca aparece como una de las categorías de análisis a las que Guamán Poma dedica su extensa obra. A lo largo del texto encontramos secciones sobre españoles, amerindios y una breve sección sobre los africanos. No existe, sin embargo, tal delimitación sistemática ni en torno a la cuestión del mestizaje en general, ni en relación a la los mestizos en particular. De hecho, como sugiere Juan Carlos Estensoro Fuchs (2000),

Huaman Poma no representa el mestizaje ni se preocupa de precisar sus variantes, aunque su texto muestra que las conoce bien y que muchos de los términos que se emplearán en el siglo XVIII ya se encontraban fijados para entonces. Si el autor no describe el mestizaje de forma sistemática es, simple y llanamente, porque para él no debería existir. (p. 69).

Ciertamente, los extensos procesos de mestizaje que estaban ocurriendo durante la época en que escribe Guamán Poma -y de los cuales estaba bien enterado como sugiere su uso de términos altamente especificativos como zambaigo y cholo- no eran parte de las memorias que quería preservar por medio de su escritura. Al contrario, el mestizaje es un hecho que Guamán Poma hubiera preferido renegar. Uno de los únicos obstáculos que imposibilitan que el autor evada por completo esta cuestión es su incesante preocupación con la proliferación de mestizos, un asunto al cual retorna una y otra vez a lo largo de El primer nueva corónica y buen gobierno.

En vez de incluir a los mestizos como una de las categorías constituyentes de su minuciosa representación de la sociedad colonial, los comentarios de

1 A lo largo del texto utilizo el término "abyecto/abyecta" no sólo para evocar la idea de algo detestable y extremamente vil, sino también en el sentido que Julia Kristeva y Judith Butler le confieren a esta palabra como aquello que cuestiona toda noción de fronteras estables y cuya exclusión es necesaria para la constitución de la identidad del sujeto. Por un lado, Kristeva (2002) arguye que "It is thus not lack of cleanliness or health that causes abjection but what disturbs identity, system, order. What does not respect borders, positions, rules. The in-between, the ambiguous, the composite" (p. 232). Butler (1993), por otro lado, explica que "This exclusionary matrix by which subjects are formed thus requires the simultaneous production of abject beings, those who are not yet 'subjects,' but who form the constitutive outside to the domain of the subject" (3). Puntualiza además que "Ajection (in latin, ab-jicere) literally means cast off, away, or out and, hence, presupposes and produces a domain of agency from which it is differentiated.... the notion of abjection designates a degraded or cast out status within the terms of sociality.... abject zones within sociality also deliver this threat, constituting zones of uninhabitalbility" (Butler, p. 243). 
Guamán Poma sobre este grupo aparecen de manera dispersa y desorganizada. Esta estrategia es consistente con el argumento de Estenssoro Fuchs e indica que la propia estructura del texto es utilizada para reforzar su contenido. Es decir, el hecho de no adjudicarle a los mestizos un espacio determinado dentro su carta-crónica refuerza la idea de que Guamán Poma busca evitar que el contexto de mestizaje en el que habita se inscriba en su texto como un aspecto inevitablemente constitutivo de la sociedad colonial a la que busca reformar. Más bien, las observaciones que Guamán Poma hace sobre este grupo aparecen entrelazadas con dos propuestas concretas que presenta como parte de las reformas coloniales que propone, y cuyo último fin es repudiar la reproducción entre indígenas y españoles -en otras palabras, la proliferación de mestizos. La primera de estas propuestas gira en torno a la producción del espacio colonial y evidencia la forma en que el modelo dual de repúblicas es problematizado, si no del todo socavado, por el avance del mestizaje. Esta cuestión no sólo no escapa al escrutinio intelectual de Guamán Poma, es precisamente lo que ocasiona su primera meditación detenida sobre la figura del mestizo.

En el primer fragmento en que Guamán Poma discursa de manera sustancial sobre los mestizos, asienta algunas de las bases argumentales a las que recurrirá repetidamente a lo largo del texto. Escribe:

para el seruicio de la coro[na] rreal de su Magestad, de nuestro señor rrey don Felipe el terzero, monarca del mundo, aumente yndios y se rredusca en sus pueblos y multiplique yndios. Y deje de multiplicar mestisos, cholos, mulatos, sanbahigos, cin provecho de la corona rreal; antes para mal que bien salen casta de biquna [vicuña] y de taruga [venado de altura], que no sale del padre ni de la madre, mala gente en gran daño de la corona rreal y de los pobres yndios deste rreyno. (Guamán Poma de Ayala, p. 215).

Aquí, Guamán Poma comienza simplemente aseverando que la población amerindia necesita aumentar. En este pasaje asocia la implementación de las reducciones toledanas con el crecimiento de la población indígena y, por tanto, presenta este aspecto de la reorganización del espacio colonial como algo positivo. ${ }^{2}$ Su estrategia retórica principal es entrelazar su propio

2 La política de reducciones tipifica en el caso andino la implementación del modelo dual de repúblicas; es decir, la separación residencial entre las repúblicas india y española. Como explica Alejandro Málaga Medina, inmediatamente después de la conquista de Perú (en 1532), hubo pocos esfuerzos por congregar a los amerindios en pueblos de indios. Tanto el Marqués de Cañete como el Conde de Nieva intentaron implementar esta política, pero se enfrentaron a una fuerte oposición por parte de los encomenderos (Málaga Medina, p. 170). En su texto, Guamán Poma alaba la figura del Marqués de Cañete (ver, por ejemplo, pp. 441, 469). Fue el virrey Francisco Toledo quien finalmente logró lo que sus predecesores no pudieron. Entre 1570 y 1575 los amerindios fueron congregados en pueblos de indios-recurriendo para esto (como señala Guamán Poma) a medidas severas como la quema y destrucción de los pueblos 
interés por la preservación de la población indígena con los intereses socioeconómicos y religiosos de la corona española. Apela a los intereses coloniales argumentando que la reproducción de la población indígena es un servicio a la Corona, puesto que, como aclara más adelante, si el número de amerindios continuara a disminuir el rey perdería su reino y, con él, no sólo derrocharía una fuente valiosa de mano de obra y de tributo perdería además la justificación ideológica de la conquista -esto es, la misión de cristianizar a los indígenas (Guamán Poma de Ayala, p. 589). Notablemente, Guamán Poma también afirma que no es provechoso a la corona que los mestizos y otras castas continúen reproduciéndose. De hecho, en sus propias palabras, "es en gran daño de la corona." La manera en la que desarrolla esta parte de su argumento es una indicación reveladora de la lógica que estructura su visión sobre la figura del mestizo.

Para empezar, como explican Adorno y John Murra en una nota aclaratoria sobre el fragmento en cuestión, Guamán Poma utiliza la imagen de dos tipos de animales no-domesticados (la vicuña y la taruga) para simbolizar a las identidades socio-raciales que critica (Guamán Poma de Ayala, p. 1135). Al hacer esta comparación, no sólo animaliza a los mestizos y demás castas mencionadas, apela a la noción de lo indomesticado para caracterizar a estos grupos y exhortar a las autoridades coloniales a que desalienten su reproducción. Desde su perspectiva, la indeterminación espacial de estas identidades dentro del modelo dual de repúblicas, coloca a estos grupos fuera del modelo de socialización operante. Y es justamente esta falta de pertenencia -o “domesticación”- lo que justifica que Guamán Poma declare que la continua reproducción de estos grupos es de poco interés socio-económico para la Corona. Esta aserción, además, sugiere que el autor busca intervenir en el tipo de discusiones que se estaban sucintando sobre estos grupos en cuanto a cuestiones como la vagancia y el tributo. Por un lado, como afirma Magnus Mörner (1970), a lo largo del siglo dieciséis la inmensa mayoría de mestizos eran considerados ilegítimos y tendían hacía la marginalidad social y la vagancia -particularmente a medida que se intentó consolidar la política de separación residencial entre las repúblicas india y española (105). Está asociación con el desarraigo espacial, según María Elena Martínez (2000), llevó a que los mestizos y otras castas se convirtieran en los principales blancos de discursos y políticas segregacionistas (13). Por otro lado, como aclara Ronaldo

anteriormente ocupados por grupos indígenas, así como la reubicación forzada (Málaga Medina 179). Además, como sugiere Monica Morales, las reducciones representan una metáfora espacial de control, pues implicaron, como arguye "the transformation of Andean people into Indians, [that is] docile bodies and subjects of virtue and production.... The reducciones stood for a model of the civilized" (Morales 109). 
Escobedo Mansilla (1981), la cuestión de tributo en zambaigos y mulatos era un asunto altamente complejo ya que, muchas veces, estos grupos no tenían lugares estables de residencia- lo cual complicaba la recaudación de tributos. En el caso de los zambaigos, además, las autoridades coloniales debatieron repetidamente qué tipo de tributo debería pagar ese grupo: el tributo que se exigía de la población indígena, o el que se demandaba de los africanos (Escobedo Mansilla 45-46). En este último caso, parte del problema radicaba precisamente en la herencia mixta (o mestiza) de los zambaigos. Al aludir a estas cuestiones, Guamán Poma busca establecer un claro contraste entre los beneficios de alentar el crecimiento de la población indígena y los prejuicios de hacer lo mismo para los mestizos y otras castas. Para él, el posicionamiento ambiguo de los individuos de herencia mestiza es un factor crucial en la desestabilización del orden del modelo dual de repúblicas, ya que cuestiona la premisa binaria de la sociedad en la que tal sistema se fundaba. La figura del mestizo, por tanto, comienza a asociarse en su texto con aquello que obstaculiza el orden y desestabiliza la noción de fronteras estables. Comienza pues a figurarse como lo abyecto.

A medida que Guamán Poma desarrolla sus comentarios sobre mestizos, se enfoca en la segregación del espacio como una de las principales medidas alrededor de las cuales organiza sus propuestas de reformas coloniales. Como sugiere Estenssoro Fuchs, si fuera decisión suya, los españoles, amerindios y africanos residirían respectivamente en Castilla, las Américas y África (p. 69). Ante la posibilidad de volver atrás, sin embargo, Guamán Poma sugiere en vez la ordenada segregación del espacio. Sobre todo, su preocupación central es mantener a los españoles, negros, mestizos y otras castas fuera de los pueblos de indios. Para validar su insistencia en estas medidas segregacionistas, Guamán Poma recurre a decretos reales que prohibían la residencia permanente de grupos no indígenas en pueblos de indios. Menciona, por ejemplo, las ordenanzas de Francisco de Toledo cuando afirma que "ni entrase ni español ni mestizo ni mulato ni negro en sus pueblo ni tierras [de indios]..., cino que fuesen a las ciudades a ueuir ellos” (Guamán Poma de Ayala, p. 448). Además de asegurar que su propuesta es consistente con la política dual de repúblicas, Guamán Poma ofrece una razón adicional para reforzar su insistencia en que los grupos estipulados no entren en las comunidades indígenas. Como dice "Ip]orque no rresultase daños y males entre yndios, que dellos rresulatría alsamiento y se enseñarían malos uicios" (ibid). Aquí, no sólo menciona la influencia negativa que estos grupos podrían tener en el comportamiento de los indígenas (diciendo por ejemplo que puede llevar a levantamientos, un punto que sigue desarrollando dada su vital importancia para las autoridades coloniales), también insiste en que aprendería e imitarían los malos vicios del 
resto de la sociedad colonial. Vemos aquí, por tanto, lo que Mónica Morales (2012) caracteriza como la instrumentalización del espacio en la obra de Guamán Poma, o sea el hecho de atribuirle a la producción del espacio un papel fundamental en la formación del carácter y comportamiento de un grupo y una cultura (104). En este caso, la instrumentalización del espacio está ligada a la cuestión de inclusión y exclusión de los pueblos de indios, pues es precisamente la posibilidad de que los indígenas imiten el mal ejemplo de los otros grupos socio-raciales lo que se busca evitar por medio de la segregación. Como indican sus comentarios, la visión de Guamán Poma de una sociedad bien organizada gira en torno a su petición de un espacio exclusivamente indígena. Su punto de partida son lugares concretos (los pueblos de indios), pero su modelo de organización socio-espacial está basada en la creación de una frontera que conceptualiza tanto en términos geográficos, como socio-raciales. Es decir, ya que la estrategia propuesta por Guamán Poma para patrullar las fronteras de pueblos de indios está basada en la habilidad de negar la entrada a todas las personas no-indígenas, es claro que su concepción del espacio va más allá de lo geográfico al abarcar también una noción de exclusividad socio-racial. ${ }^{3}$

La visión de Guamán Poma sobre la segregación del espacio se reitera repetidamente a lo largo de su carta-crónica. Mas a medida en que avanzan sus puntualizaciones sobre esta cuestión, su preocupación por la proliferación de mestizos lo lleva también a ahondar en la temática que subyace detrás de este fenómeno: es decir, las relaciones sexuales entre amerindios(as) y españoles(as). Escribe:

Ci fuere española o mestiza o mulata que fuere casado con yndio en este rreyno, que haga los dichos españoles sus pueblos fuera de los yndios en una jornada, ya que no fuera de una legua. Y ci pariere mestiza, chola, lo lleue a la ciudad por el escándalo de ellas. (Guamán Poma de Ayala, p. 547).

En este caso agrega que si un indígena llegara a casarse con alguien fuera de su grupo socio-racial, ese hombre y su mujer deberían ser excluidos de lo que para él es una visión muy particular sobre comunidades indígenas perfectamente herméticas. Especifica la distancia mínima que se debe tener entre los pueblos de indios y los lugares de residencia donde el resto de la

3 Al apelar a la noción de raza como una categoría de análisis válida para el periodo en cuestión, me apoyo en estudios recientes cuya meta es historizar este constructo cultural para mejor teorizarlo. Estos estudios incluyen:

Genealogical Fictions: Limpieza de Sangre, Religion, and Gender in Colonial Mexico (2008) por María Elena Martínez; Racisms: From the Crusades to the Twentieth Century (2013) por Francisco Bethencourt; Racism: A Short History (2002), por George M. Fredrickson; Racist Culture (1993) por David Theo Goldberg; y "Purity of Blood. Problems of Interpretation” (2012) por Max S. Hering Torres. 
población (mestiza o no) debería habitar, y enfatiza el escándalo de concebir un hijo mestizo dentro de un pueblo de indios, al mismo tiempo en que insiste en que ese hijo debe también ser excluido de la comunidad. En este caso, Guamán Poma va más allá de las ordenanzas toledanas ya que, según se estipula en el Libro VI, Título III: "y en quanto á los Mestizos, y Zambaygos, que son hijos de Indias, nacidos entre ellos, y han de heredar sus casas, y haciendas, porque parece cosa dura separarlos de sus padres, se podrá dispensar" (Recopilación de Leyes de los Reynos de las Indias, 1791, p. 212). ${ }^{4} \mathrm{Al}$ contravenir esta dispensación, la postura de Guamán Poma indica que su visión va más allá de un interés por patrullar las fronteras externas de los pueblos de indios. Utiliza esta misma lógica excluyente de manera interna una vez que comienza a estipular los casos en que amerindios pueden ser también expulsados de los pueblos de indios. Considerando que este nuevo criterio está basado en la selección matrimonial de una persona (lo cual implica tanto sexo como reproducción) comenzamos entonces a discernir la forma en que su noción de una frontera geográfica entre amerindios y el resto de la población colonial está inextricablemente vinculada con el reforzamiento de una frontera etno-sexual. ${ }^{5}$

Una segunda serie de propuestas alrededor de las cuales Guamán Poma organiza sus comentarios sobre mestizos gira en torno a la necesidad de proteger la castidad femenina amerindia. A diferencia de sus planteamientos sobre organización socio-espacial -en los que tiende a agrupar a los mestizos con el resto de las identidades socio-raciales que busca excluir de los pueblos de indios- sus afirmaciones en derredor a esta segunda cuestión evidencian un enfoque exclusivo en la figura del mestizo. Su objetivo central es desalentar la continua reproducción de este grupo y, para esto, identifica a la sexualidad

4 Además, como aclara Berta Ares Queija(2000) "residir en los pueblos de indios fue algo que ... la Corona prohibió reiteradamente y en principio de manera indiscriminada a 'espanoles, negros, mestizos, mulatos y zambaigos.' Sin embargo, y aparte las continuas transgresiones, desde la década de 1570 y por disposición del virrey Toledo, los corregidores podín consentírselo a aquellos españoles que no 'perjudicasen' a los indios y también a los mestizos y zambaigos que vivían entre sus familiares, pero no a negros y mulatos libres.” (86).

5 Como sugieren los críticos Abdul JanMohamed (1993), Joane Nagel (2000) y Robert Bernasconi (2012), la noción de una frontera racial implica siempre/ya la noción de una frontera sexual. JanMohamed introduce el término "sexualidad racializada"(o racialized sexuality) precisamente para definir la transgresión de esta doble frontera construida socio-jurídicamente (pp. 104,109). Nagel arguye que las fronteras raciales (que para ella son siempre también fronteras sexuales) son constantemente transgredidas por relaciones eróticas, pero a la vez son constantemente patrulladas y protegidas (pp. 107, 113). Bernasconi, por su parte, argumenta que la noción de raza opera como un concepto de frontera cuya existencia está predicada en la creación de fronteras (las cuales entiende tanto en términos geográficos como sexuales) pero cuya operación nunca depende por completo de los esencialismos que se busca establecer por medio de estas fronteras (pp. 207-208, 213). 
femenina indígena como un ámbito crucial de intervención. Al articular esta parte de su argumento, Guamán Poma oscila ambivalentemente entre presentar a las mujeres amerindias como seres pasivos que necesitan ser protegidos y caracterizarlas como agentes cómplices de su propia explotación sexual. Si bien esta serie de cometarios evidencian un inquebrantable anhelo por proteger y estimular el crecimiento de la población indígena, es evidente también que para el autor este objetivo es indisociable de un ímpetu paralelo: el de frenar la proliferación de mestizos.

El segundo comentario extendido sobre mestizos que encontramos en $E l$ primer nueva corónica y buen gobierno se deriva de una sección en la que Guamán Poma lamenta la pérdida de leyes y costumbres que regulaban la castidad de las mujeres indígenas. Tras discurrir sobre la forma en que doncellas vírgenes eran respetadas y valoradas antes de la llegada de los españoles, agrega "[t]odo lo malo adulterio y otros pecados mortales trajo concigo los dichos cristianos; con color de la doctrina deruirga a todas la donzellas y aní [sic] paren muchos mestisos en este rreyno”(Guamán Poma de Ayala, p. 226). Aquí, Guamán Poma imputa moralmente a los españoles y asevera que son ellos los responsables de introducir el adulterio y otros pecados mortales a la sociedad andina. Si bien esto no quiere decir que esté sugiriendo que el adulterio no existía antes de la conquista, como aclara más adelante de forma textual y visual, el adulterio y las relaciones sexuales pre-maritales eran crímenes severamente punidos antes de la imposición del orden español (Guamán Poma de Ayala, pp. 308-311). Como afirma Alejandra Osorio (1990), la violación de la virginidad amerindia es presentada en este y otros pasajes como "el paradigma central de la catástrofe social -'el mundo al revés'- que trajo la conquista para los pueblos andinos” (294). En la cita, Guamán Poma además introduce una de sus más tenaces críticas: su aserción de que la doctrina cristiana ha sido utilizada para justificar el desfloramiento de inúmeras mujeres indígenas. Es altamente significativo, no obstante, que al enfatizar lo reprochable que son cada una de estas acciones, no deja de lado lo que, para él, es su igualmente negativa consecuencia: la reproducción de mestizos. Al asociar los excesos sexuales de la conquista con el aumento de mestizos, Guamán Poma desplaza sus críticas sobre este grupo hacia un plano moral y comienza a desarrollar una lógica argumental fundamentada en un dúo interés. Por un lado, el de denunciar la persistencia de abusos sexuales por parte de las autoridades coloniales y, por otro, el de establecer una correlación entre la continuidad de estas injusticias y el nacimiento de mestizos.

Más allá de los abusos sexuales asociados con la conquista, Guamán Poma denuncia abiertamente la ininterrumpida violación de mujeres indígenas por parte de corregidores, encomenderos, padres y otras autoridades coloniales 
(pp. 232, 448, 508). En particular, se enfoca en los padres católicos para resaltar el hecho de que dichas injusticias continúan ocurriendo bajo el régimen colonial español. Se queja repetidamente de que los padres les roban las esposas a los amerindios y que desviran a las mujeres solteras (448); asevera que algunos padres se visten como amerindios para entrar a sus casas y poder ver a las mujeres desnudas (o como él dice "se la mira la güergüienza" (508); y denuncia el hecho de que muchos padres cohabitan con mujeres indígenas a pesar de sus votos de castidad (580). Sin restarle importancia al lugar que este tipo de denuncias tienen dentro de la visión de justicia a la que apela interminablemente Guamán Poma, es revelador que el recuento de cada uno de estos abusos está inevitablemente entrelazado con lo que para él es la igualmente perniciosa reproducción de mestizos. Esta correlación es confirmada por medio de una de las tres ilustraciones en las que Guamán Poma incluye visualmente a la figura del mestizo. ${ }^{6}$

La ilustración es parte de una sección que Guamán Poma dedicada a los padres católicos y aparece bajo el encabezado "Hijo de los padres dotrinantes mesticillos y mesticil[l]as / Lo lleua un harriero español alquilado a la ciudad de los Reys de Lima, media dozena de niños lo lleua / doctrina" (620). La escena incluye a dos hombres adultos, uno a pie que tira de las riendas de un caballo cargado de media decena de infantes mestizos(as), y otro montado a caballo. La imagen captura el momento en que el grupo de mestizos(as) es entregado al arriero alquilado para que éste se encargue de ocultar toda evidencia inculpatoria de que el padre no sólo ha contravenido sus votos de castidad, lo ha hecho en repetidas ocasiones. Los(as) seis mestizos(as) -producto de las relaciones sexuales entre el padre y diferentes mujeres indígenas a las que, como aclara el texto acompañante, éste desvirgó utilizando como excusa la enseñanza de la doctrina- representan el escándalo de estas uniones interraciales. Son seres abyectos que deben ser expulsados del espacio social en que nacieron y escondidos por simbolizar el quiebre del orden social. Los rasgos fisionómicos utilizados por Guamán Poma para caracterizar a este grupo de mestizos(as) son poco distintivos, y es inclusive difícil diferenciarles en términos de género -a pesar de que el texto especifica que hay tanto niños como niñas. El hecho

6 Las otras dos ilustraciones aparecen en las páginas 509 y 617. En cada una de ellas, se reúnen en la mesa del corregidor y el padre respectivamente diferentes grupos socio-raciales, incluyendo mestizos. Como arguye Estenssoro Fuchs "Esta escena hace patente para Huaman Poma el desorden absoluto, puesto que es inaceptable que la autoridad busque compartir su mesa para divertirse con 'gente baja”' (71). También sugiere que Guamán Poma evita retratar la figura del mestizo por la dificultad de "atribuirles rasgos fisionómicos estandarizados y vestimentas características" (71). Cada escena representa así un microcosmos del desorden, en la que las jerarquías socio-raciales han sido abolidas y en las que la falta de segregación hace patente el desorden absoluto. 
de que sean media docena de infantes es un tanto hiperbólico (considerando su atribución a un solo padre), pero la cantidad contribuye al factor alarmante de la escena. En la ilustración, además, dos de los(as) mestizos(as) levantan el dedo índice en señal de pedir la palabra, pero es evidente que los hombres adultos sólo se miran entre sí, ignorando del todo cualquier objeción que los(as) mestizos(as) pudieran tener. La ausencia en la escena de las madres es también significativa, pues indica que, para el autor, es el resultado de estas uniones entre padres españoles y amerindias lo que preocupa, no lo que las mujeres indígenas pudieran pensar y sentir al ser separadas de sus hijos(as). Si bien el objetivo de la ilustración es denunciar la persistencia de abusos sexuales por parte de las autoridades coloniales, lo más sobresaliente de la imagen -lo que busca escandalizar y alarmar- es el creciente número de mestizos cuyo lugar en la sociedad les es constantemente negado. Es esta indeterminación social lo que justifica una y otra vez que Guamán Poma evada la representación sistemática de este grupo y opte en vez por incluirlos en su texto como la figura de lo abyecto.

Al denunciar los continuos ultrajes de los que son víctimas las mujeres indígenas, Guamán Poma busca además resaltar la necesidad de proteger su castidad. Por un lado, comienza presentándolas como seres pasivos que precisan de la protección masculina amerindia. Para esto, especifica que una de las funciones centrales de los caciques principales dentro de los pueblos de indios debe ser la de defender a la comunidad de "padres y de españoles y caualleros y judíos y moros y mestizos y mulatos y negros" (Guamán Poma de Ayala, pp. 502). Específicamente, puntualiza que los caciques precisan evitar, entre otras cosas, que tales hombres abusen sexualmente de las mujeres amerindias; en sus palabras, deben defender en contra del "forsar de sus mugeres, hijas, donzellas" (ibid; énfasis mío). Aquí, el uso del adjetivo posesivo "sus" para calificar a las amerindias que necesitan de la protección masculina indígena implica una sumisión patriarcal y confirma la pasividad inicial utilizada para describir a estas mujeres. Guamán Poma critica además el hecho de que algunos amerindios lleven consigo a trabajar en las minas a sus hermanas e hijas -las cuales, arguye, terminan amancebadas con los mineros y mayordomos- y sugiere, en vez, que dichas mujeres no debería abandonar los pueblos de indios (536). En otras palabras, propone aislar a las mujeres indígenas de todo contacto con hombres que no sean de su mismo grupo socioracial. Así, de igual forma en que propone patrullar el espacio para excluir a grupos no-indígenas de los pueblos de indios, aquí los cuerpos de las mujeres se convierten en una especie de tropo espacial que igualmente necesita ser segregada de todo contacto con elementos no-indígenas. Para Guamán Poma, esta cuestión es literalmente de vital importancia para la preservación de la 
población amerindia y, sin embargo, es también un asunto inevitablemente vinculado con su rechazo del mestizaje. Como afirma Sara Vicuña Guegerich (2013), Guamán Poma demanda la segregación total entre indígenas y españoles, y rechaza las uniones sexuales entre estos grupos, dada su doble preocupación por el declive de la población indígena y por la proliferación del mestizaje (680). Su consistencia en este argumento, no obstante, no impide que al identificar la protección de la castidad amerindia como un factor clave para encarar estas dos problemáticas, Guamán Poma fluctúe ambivalentemente en su caracterización del rol de las mujeres en este proceso.

En contraste con su descripción de las mujeres indígenas como seres pasivos cuya sexualidad debe ser resguardada, Guamán Poma asevera que muchas amerindias se han convertido, en sus palabras, en "grandes putas" por ser participes de su propia explotación sexual (pp. 448, 508, 539, 553, 567, 579). En ocasiones, como señala Vicuña Guengerich, Guamán Poma presenta la misma violación de amerindias -a pesar de ser un acto en contra de su voluntad- como la causa de que estas mujeres se transformen en prostitutas (680). En este caso, su postura es ambivalente, pues conlleva tanto la necesidad de proteger a las mujeres, como su rechazo y admonición de éstas una vez que han sido violentadas. En dos diferentes pasajes, además, Guamán Poma reprende abiertamente a las mujeres indígenas que optan por tener relaciones exogámicas con los españoles y, dada esta selección, las califica como "grandes putas" $(553,579)$. Se queja, asimismo, de que el resultado de tales acciones es la indeseable reproducción de mestizos.

En el primero de estos fragmentos, Guamán Poma afirma que las mujeres indígenas "Ya no quiere al yndio, cino a españoles y se hazen grandes putas y paren todo mestizos, mala casta en este rreyno" (p. 553). Aquí, la preferencia sexual de la mujer indígena es lo que ocasiona el juicio moral de Guamán Poma; es lo que merita que las llame grandes putas y que las acuse de reproducir la mala casta de mestizos. Asimismo cuando escribe:

Y en ello, las yndias hazen grandes putas y no ay rremedio. Y [aci]na no se quieren casar porque ua tras del padre o del español. Y ací no multiplica yndios en este rreyno, cino mestizos y mestizas y no ay rremedio. (Guamán Poma de Ayala, p. 579).

En este pasaje, la predilección de las mujeres indígenas por los españoles es más patente, ya que éstas son presentadas como agentes que persiguen activamente el objeto de su deseo. El calificativo de prostitutas también adquiere otro matiz pues es directamente vinculado con la cuestión del matrimonio. Al afirmar que las mujeres prefieren ir detrás del los padres y otros españoles en vez de casarse, Guamán Poma contribuye a la común asociación de los mestizos con la ilegitimidad, pero incluye también un juicio 
moral en contra de las mujeres que optan por ser participes en este tipo de relaciones extramaritales. En ambos ejemplos, la habilidad de una mujer de escoger su propia pareja sexual es presentada en términos altamente negativos. Esta preferencia sexual voluntaria se convierte en un símbolo de traición. Asimismo, en ambos casos se recalca el hecho de que las relaciones exogámicas contribuyen a la reproducción de mestizos y, notablemente, se re-establece la correlación entre el aumento de éstos y la disminución de la población indígena. Al enfatizar estas cuestiones, Guamán Poma busca exhortar a las mujeres indígenas a preservar su castidad y recalca la importancia de la reproducción endogámica. La vigilancia de la sexualidad femenina amerindia se reitera, por ende, como un ámbito crucial de intervención. A pesar de que su postura oscila ambivalentemente entre presentar a las amerindias como seres pasivos que deben ser protegidos y agentes participes de su propia explotación sexual, es evidente que para Guamán Poma el rol de las mujeres en la reproducción biológica es un factor ineludible en su afán por encarar tanto la estimulación del crecimiento de la población indígena como la disuasión del aumento de la población mestiza.

El análisis de la caracterización abyecta del mestizo en El primer nueva corónica y buen gobierno nos compele a reflexionar sobre la forma en que nociones emergentes de diferencias socio-raciales irrumpen en el discurso colonial latinoamericano y van negociándose por los sujetos que vivencian el mundo en transformación en las que estas nociones se desarrollan. En la obra de Guamán Poma, la indeterminación espacial de los mestizos y otras castas dentro del modelo dual de repúblicas representa no sólo un quiebre del modelo de socialización operante en la época, evidencia también la forma en la que la perturbación de este orden se va articulando en tándem con posibles soluciones. Para el autor, parte de la solución puede encontrarse en la existente política segregacionista del espacio, pero es necesario reforzar las ordenanzas y encarar una segunda problemática. Más allá de reflexiones sobre la producción del espacio, el texto de Guamán Poma exhibe además un perspicaz interés en el tipo de relaciones reproductivas que contribuyen al crecimiento de la población mestiza. En particular, identifica a la sexualidad femenina amerindia como un ámbito crucial de intervención para frenar la reproducción de mestizos. Si bien la figura del mestizo aparece en la obra de Guamán Poma no como un componente más de su meticulosa representación de la sociedad colonial, sino más bien como la figura de lo abyecto, es evidente que esta estrategia de inclusión exclusiva es su forma de negociar el cambiante escenario de relaciones socio-raciales en las que se inscribe su texto. 
OLIMPIA E. RosenthaL

\section{Referencias bibliográficas}

ADORNO, Rolena (2000). Guaman Poma. Writing and Resistance in Colonial Peru. Austin:University of Texas Press.

ARES QUEIJA, Berta (2000). "Mestizos, mulatos y zambaigos. Virreinato del Perú, siglo XVI. ” En Berta Ares Queija y Alessandro Stella (coordinadores) Negros, Mulatos, Zambaigos. Derroteros africanos en los mundos ibéricos. Sevilla: Publicaciones de la Escuela de Estudios Hispano Americanos.

BERNASCONI, Robert (2012). "Crossed Lines in the Racialization Process: Race as a Border Concept" en Research in Phenomenology 42; pp. 206-228.

BETHENCOURT, Francisco (2013). Racisms:From the Crusades to the Twentieth Century. Princeton; Oxford: Princeton University Press.

BUTLER, Judith (1993). Bodies that Matter. On the Discursive Limits of Sex. London; New York: Routledge.

ESCOBEDO MANSILLA, Ronald (January 1981). "El tributo de los zambaigos, negros y mulatos libres en el virreinato peruano" en Revista de Indias, 41; pp. 43-54.

ESTENSSORO FUCHS, Juan Carlos (2000). "Los colores de la plebe: razón y mestizaje en el Perú colonial” en Natalia Majluf (edición) Los cuadros de mestizaje del virrey Amat y La representación etnográfica en el Perú colonial. Lima: Museo de Arte de Lima; pp. 66-107.

FREDRICKSON, George M (2002). Racism: A Short History. Princeton, NJ; Oxford: Princeton University Press.

GOLBERG, David Theo (1993). Racist Culture. Cambridge, Mass.: Blackwell.

GUAMÁN POMA DE AYALA, Felipe (2006) [1615/1616]. El primer nueva corónica y buen gobierno. Cuarta Ed. John V. Murra and Rolena Adorno, eds. México: Siglo Veintiuno.

HERING TORRES, Max S (2012). "Purity of Blood. Problems of Interpretation" en Max S. Hering Torres, María Elena Martínez y David Niremberg (editores) Race and Blood in the Iberian World. Berlin: Lit Verlag; pp. 11-38.

JANMOHAMED, Abdul (1993). "Sexuality on/of the Racial Border: Foucault, Wright, and the Articulation of 'Racialized Sexuality"' Domma Stanton (editor) en Discourses of Sexuality: from Aristotle to AIDS.Ann Arbor: University of Michigan Press; pp. 94-117.

KRISTEVA, Julia. "Powers of Horror." en Kelly Oliver (editor) The Portable Kristeva. New York: Columbia University Press; pp. 229-263.

MÁLAGA MEDINA, Alejandro (July-December 1979). "Aspecto Urbano de las Reducciones Toledanas" en Revista de Historia de América, 88; pp. 167-183.

MARTÍNEZ, María Elena (2008). Genealogical Fictions: Limpieza de Sangre, Religion, and Gender in Colonial Mexico. Stanford: Stanford University Press. 
MARTÍNEZ, María Elena (2000). "Space, Order, and Group Identities in a Spanish Colonial Town: Puebla de los Angeles" en Luis Roniger and Tamar Herzog (editores) The Collective and the Public in Latin America: Cultural Identities and Political Order. Brighton; Portland: Sussex Academic Press; pp. 13-36.

MORALES, Mónica P (2012). Reading inebriation in early colonial Peru. Farnharm, Surrey;Burlington, VT: Ashgate.

MÖRNER, Magnus (1970). La corona Española y los foráneos en los pueblos de indios de América. Estocolmo: Almqvist \& Wiksell.

NAGEL, Joane (2000). "Ethnicity and Sexuality" en Annual Review of Sociology, 26; pp. 107-133.

Recopilación de Leyes de los Reynos de las Indias, Mandadas Imprimir y Publicar por la Magestad Católica del Rey Don Carlos II Nuestro Señor (1791). Volumen 2, Título III, Libro VI.

OSORIO, Alejandra (1990). "Seducción y conquista: Una lectura de Guamán Poma” en Allpanchis 22, 35-36; pp. 293-327.

VICUÑA GUENGERICH, Sara (December 2013). "Virtuosas y corruptas: Las mujeres indígenas en las obras de Guamán Poma de Ayala y el Inca Garcilaso de la Vega" en Hispania. 96, 4; pp. 672-683. 
